\title{
Detection of Organic Colorants in Historical Painting Layers Using UV Laser Ablation Surface-Enhanced Raman Microspectroscopy***
}

\author{
Anna Cesaratto, Marco Leona,* John R. Lombardi, Daniela Comelli, Austin Nevin, and \\ Pablo Londero*
}

\begin{abstract}
Surface-enhanced Raman spectroscopy (SERS) has been increasingly used in the study of works of art to identify organic pigments and dyes in paintings, which (depending on the material) are difficult or not possible to detect by other current methods. The application of SERS to the study of paintings has been limited, however, by the lack of a sampling approach with sufficient sensitivity and spatial resolution. We show that ultraviolet laser ablation (LA) sampling coupled with SERS detection can be successfully used to study paint layers. LA-SERS permitted the isolation of signals from colorants in individual thin paint layers in sample crosssections, avoiding contamination from adjacent layers. These results expand the range of analytical applications of SERS demonstrating how the technique can be used to sensitively detect minor organic components in complex matrices. While this is fundamental for the study of cultural heritage, it is also relevant in other fields such as forensic analysis, food science, and pharmacology.
\end{abstract}

In the last decade, surface-enhanced Raman spectroscopy (SERS) ${ }^{[1]}$ emerged as an important and uniquely sensitive technique for the analysis of organic colorants in works of art and other cultural heritage material. ${ }^{[2]}$ Organic colorants, used as textile dyes or as lake pigments (that is, complexes of an organic colorant with a polyvalent metallic ion) in paintings, illuminated manuscripts, or polychrome sculptures are of relevance as indicators of historical practice and as provenance markers, but they usually are difficult to identify because of their typically low concentration. Detection is further complicated because of the heterogeneous nature of

[*] Dr. M. Leona, Dr. P. Londero

Department of Scientific Research

The Metropolitan Museum of Art, New York NY (USA)

Marco.Leona@metmuseum.org

E-mail: Pablo.Londero@metmuseum.org

A. Cesaratto, Dr. D. Comelli

Dipartimento di Fisica

Politecnico di Milano, Milano (Italy)

Prof. J. R. Lombardi

Department of Chemistry and Center for Analysis of Structures and Interfaces (CASI)

The City College of New York, New York, NY (USA)

Dr. A. Nevin

Institute of Photonics and Nanotecnologies-Consiglio Nazionale, delle Ricerche (IFN-CNR) (Italy)

$[* *$ We are indebted to the National Science Foundation (Grant CHE works of art: the colorant is present in minor or trace concentration in a complex matrix (binders, extenders, substrates such as wood or textile fibers). Much of the success of SERS in the field of cultural heritage to date is due to its tremendous sensitivity and commensurately small sample requirements. Different procedures have been developed, ${ }^{[2, d, 3]}$ generally relying on silver or gold colloids in water. This approach is simple and cost effective, but it is generally limited to the study of water-soluble compounds. Spatial resolution is another concern, as this parameter is essentially limited to about $25-50 \mu \mathrm{m}$ by the size of the sample that can be removed with scalpels, and/or the smallest amount of colloid that can be delivered to the sample. For a sample as complex as a cross-section from a painting, spatial resolution in the order of $10 \mu \mathrm{m}$ or better is essential to analyze individual layers or single features within a layer.

Several SERS-based approaches have attempted to maximize sensitivity and spatial resolution, with the aim of providing improved analysis of paintings. Gel-based SERS active substrates produce virtually no mechanical damage and have been applied directly on painting surfaces ${ }^{[4]}$ without the need to sample the work of art. The most successful highresolution implementations have used mechanical removal of paint layer microsamples with a maximum resolution of approximately $20 \mu \mathrm{m}$ typical of the most current sampling techniques. ${ }^{[5]}$ This is still insufficient in the case of multilayered microsamples because finer spatial resolution is often needed for the thinner paint layers that can often be $5 \mu \mathrm{m}$ thick or less, or for samples with fine granular structure.

In this work we demonstrate a novel SERS-based technique which combines minimal sampling requirements with spatial resolution as high as $13 \mu \mathrm{m}$. This is, to our knowledge, the smallest demonstrated spatial resolution achieved in SERS-based studies of samples from actual paintings. The instrument employs ultraviolet (UV) laser ablation in the desorption regime to precisely control the diameter and depth of the sampling region, followed by invacuum surface-enhanced Raman detection. In a prior embodiment of this laser ablation surface-enhanced Raman spectroscopy (LA-SERS) technique, ${ }^{[6]}$ sampling was achieved by tuning the laser in the visible at the chromophore resonance of the dye of interest, enabling the selective desorption of non-homogeneous samples. As we will show, UV-based desorption ${ }^{[7]}$ yields the robust and uniform heating required for microsampling of complex organic mixtures by targeted vaporization, and at the same time provides more control over the quantity of ablated material than traditional approaches. Minimizing the impact of analysis, even when the measurement is performed on a sample already removed 
from a work of art, can be essential: paintings crosssections are generally preserved for sequential and repeated analysis and archived as extremely valuable documents.

A procedure for both the pretreatment and the analysis steps was developed and characterized through a multianalytical approach, with the goal of maximizing signal sensitivity while minimizing sample damage induced by the treatment.

Our prior works show that briefly exposing a sample containing lake pigments to HF vapor can increase the sensitivity of the SERS analysis, whereas it produces only little visual alteration to the underlying substrate. ${ }^{[2 \mathrm{~d}, 8]}$ The acid vapor hydrolyzes the lake, breaking the molecular bonds between the mordant metal ion and the charged group in the dye molecules. To improve the method and reach the best balance between increased hydrolysis (and thus better analyte signal) and limited changes to the sample as a whole, the effect of increasing exposure times to HF was tested on a reference sample simulating a typical painting cross-section.

After each exposure time, the sample was ablated and the SERS spectra were collected. Once the best exposure time and ablation conditions (laser power and number of pulses) were identified, we also measured the diameter and depth of the ablated crater and the penetration of fluoride into the sample. The reference sample was taken from a tempera painted board prepared in the laboratory according to traditional practice. The cross-section is composed of lac lake particles mixed with lead white and whole egg binder. The optical set up was tuned to give an ablation size of around $25 \mu \mathrm{m}$ in diameter. The SERS spectra were collected after $0 \mathrm{~s}, 30 \mathrm{~s}, 1 \mathrm{~min}, 2 \mathrm{~min}, 4 \mathrm{~min}$, and $6 \mathrm{~min}$ of exposure to HF vapor. No signal was detect without the pretreatment step, and an increase in the intensity of the $1406 \mathrm{~cm}^{-1}$ lac lake SERS band (ascribable to $\mathrm{C}-\mathrm{C}$ stretching/ $\mathrm{C}-\mathrm{H}$ in plane bending $^{[2 a]}$ ) was noticed as a function of the exposure time with a leveling after $4 \mathrm{~min}$, as shown in Figure $1 b$.

An atomic force microscopy (AFM) scan allowed us to evaluate the dimension of the ablated crater and ascertain the invasiveness of the technique. The crater was estimated to be approximately $20 \mu \mathrm{m}$ large and $1.5 \mu \mathrm{m}$ deep. To characterize the penetration of HF below the surface of a sample, fluorine ions were mapped on the perpendicularly cut cross-section with scanning electron microscopy energy-dispersive X-ray spectroscopy (SEM-EDS). As shown in Figure $1 d$, the fluorine concentration is homogeneous all over the cross-section. Nevertheless, the aforementioned robustness of bulk samples to HF vapor hydrolysis suggests that it should be possible to apply such a pretreatment with similar parameters variations to cross-sections taken from a)
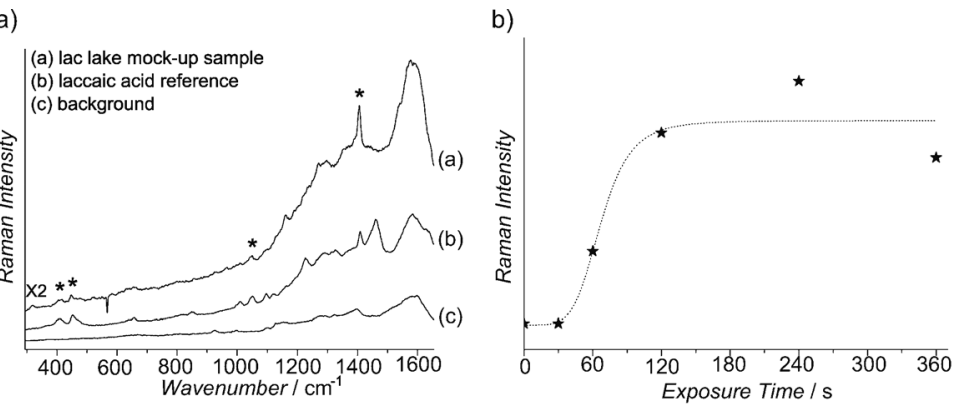

c)

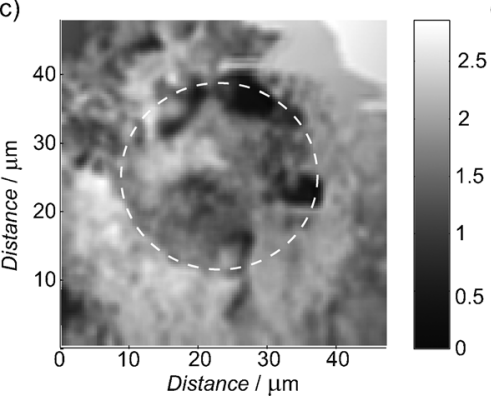

d)
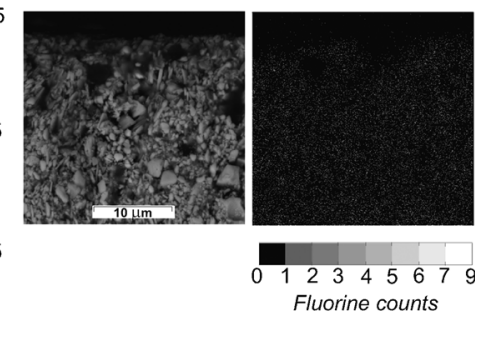

Figure 1. a) UV-LA-SERS spectrum of the lac lake mock-up sample after 4 min exposure time. Signals of reference laccaic acid were detected at $406 \mathrm{w} \mathrm{cm}^{-1}$, $453 \mathrm{w} \mathrm{cm}^{-1}, 1050 \mathrm{vw} \mathrm{cm} \mathrm{cm}^{-1}$, and $1406 \mathrm{~s} \mathrm{~cm}^{-1}$ and highlighted in the graph with an asterisk (vw very weak, $\mathrm{w}$ weak, $\mathrm{m}$ medium, $\mathrm{s}$ strong, vs very strong). The background signal is reported with a black line (c). b) The Raman intensity of the $1406 \mathrm{~cm}^{-1}$ band as a function of the HF vapor exposure time. c) AFM image of the ablated crater. d) SEM-EDX map of the HF penetration.

a)
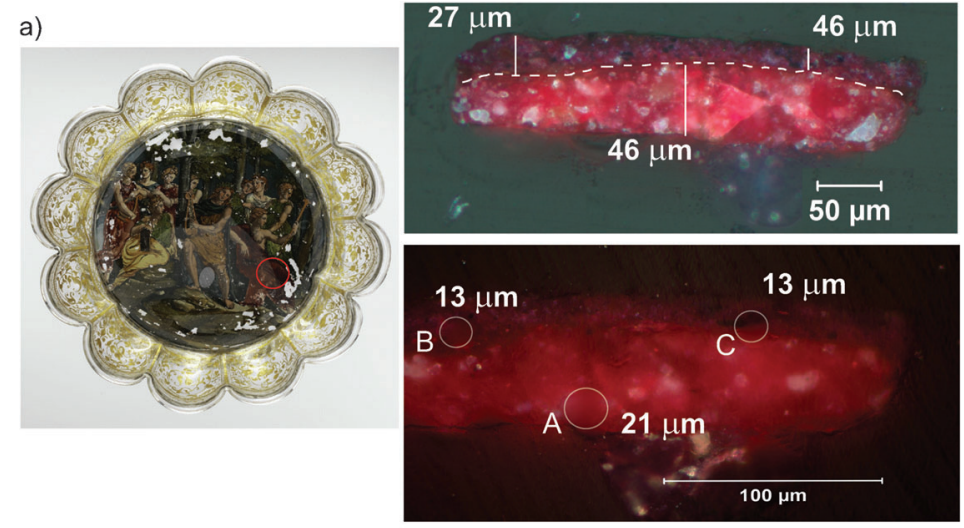

b)

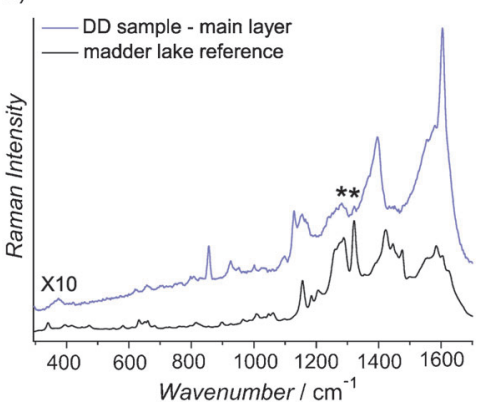

c)

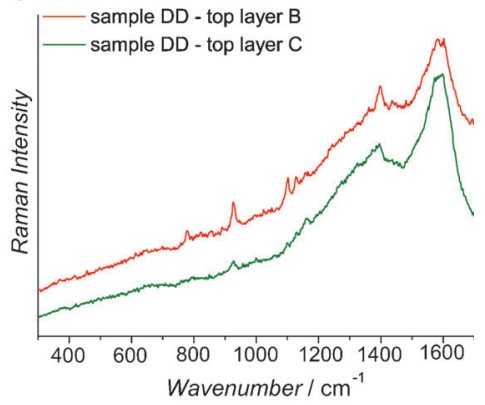

Figure 2. a) Picture of the decorated dish and microscope images of the sample $\mathrm{DD}$, with the ablated crater A, B, and C. b) SERS spectrum of the UV-ablated crater $A$ in the main layer. Signals of reference madder lake were detected at $1283 \mathrm{w} \mathrm{cm}^{-1}$ and $1321 \mathrm{w} \mathrm{cm} \mathrm{cm}^{-1}$ and highlighted in the graph with an asterisk. c) SERS spectra of the UV-ablated craters B and C in the top layer. 
real works of art. In any case, depending on the potential materials involved, adverse reaction must be considered and microtoming may be necessary. The standardized procedure ( 4 min HF pretreatment, 2-3 laser pulses, $\approx 1 \mathrm{~J} \mathrm{~cm}^{-2}$ ) can produce clear characterizing spectra while impacting only the first few microns of material below the surface, preserving the sample for further studies.

As seen from the SERS spectra in Figure $1 \mathrm{a}$, a significant background signal is present. Since the concentration of the plume of the ablated material and the signal level reduces quadratically as a function of the distance, ${ }^{[6]}$ the SERS peaks are expected to decrease too. This property was used to check the consistency of the marker peaks. In all the graphs reported, the peaks used for the identification of the organic colorants are marked with an asterisk on the top, whereas the background peaks are marked with a circle above.

UV-LA-SERS was applied to several paint cross-sections taken from original works of art, as listed in Table 1. Samples were chosen that contain features which demonstrate the potential of this technique, particularly the high spatial resolution.

The sample DD was taken from the red area of a 16th century decorated dish (Figure 2). The cross-section presents two superimposed red layers. The top layer is composed of sparse carbon particle embedded in a red glaze, the bottom one is a lighter red glaze.

Sample DD was ablated in three different areas (highlighted in Figure $2 \mathrm{a}$ ), with a minimum spot size analysis about $13 \mu \mathrm{m}$ wide. The high resolution allowed us not only to characterize the two different layers separately and without contaminating one sample with the other, but also to distinguish between carbon particles and red glaze in the very top thin layer. This is particularly critical given graphite's ability to displace colorants on the SERS substrate, which can result in it being the only detectable component. The bottom layer can be ascribed to a madder-based lake, whereas the upper one gave the SERS spectra of an unknown material. The results reinforced suspicions of a modern addition, suspected from previous visual inspection of the object.

The improved spatial resolution of UV-LA-SERS was also critical for the analysis of microsamples taken from the paintings of two Venetian masters. The sample taken from the fresco "The incredulity of San Thomas" by Luca Signorelli (sample S) required high spatial resolution for the analysis of the red glaze in the top layer, due to the reduced thickness, as shown in Figure 3 a.

UV-LA-SERS spectra gave a good match with a kermesic acid lake (corresponding to the insect dye kermes) as shown in Figure 3b. A loose sample taken from the same area of the painting was successfully analyzed with the standard colloid technique ${ }^{[9]}$ but UV-LA gave a better result, as can be seen in Figure $3 \mathrm{c}$. This is probably due to the high spatial resolution of UV-LA-SERS allowing us to focus on the red glaze, avoiding interference from the other layers. a)

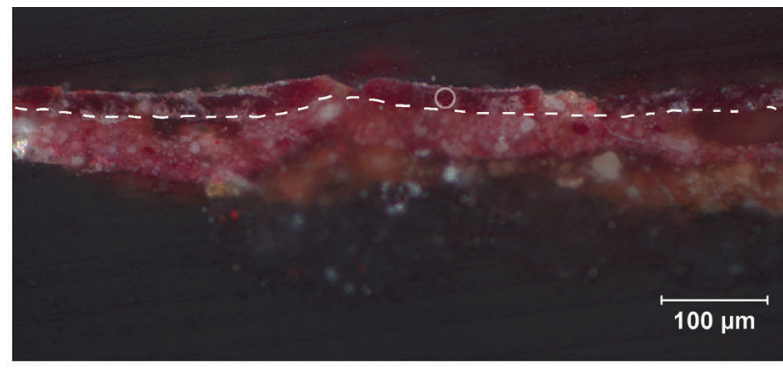

b)

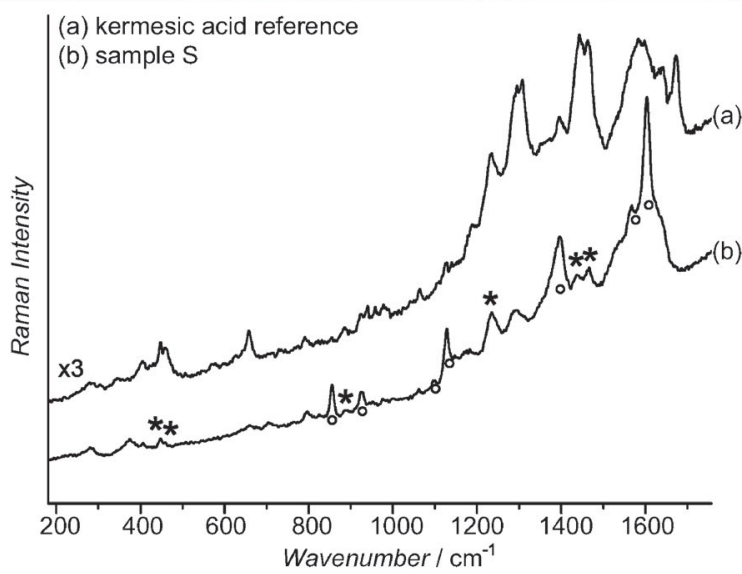

c)

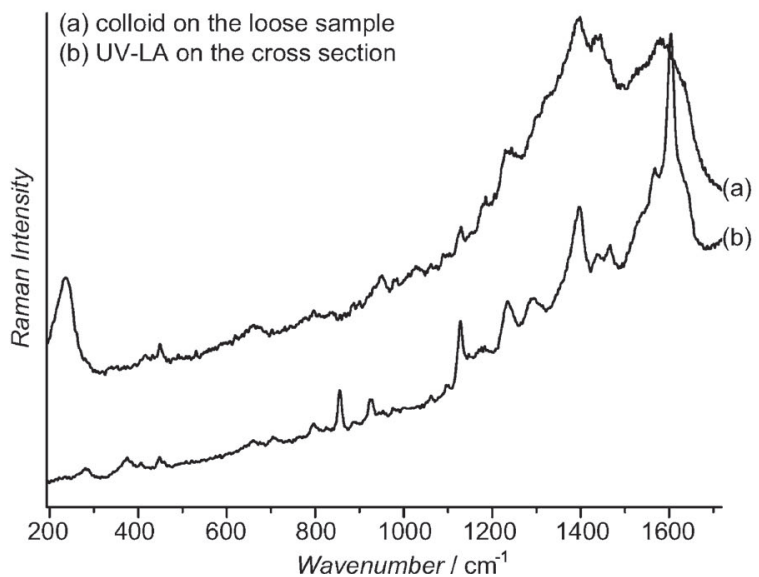

Figure 3. a) Microscopy image of the cross-section $\mathrm{S}$ with the ablated crater. b) SERS spectra obtained with UV-ablation. Signals of reference kermesic acid were detected at $447 \mathrm{w} \mathrm{cm}^{-1}, 459 \mathrm{vw} \mathrm{cm}^{-1}, 885 \mathrm{vw} \mathrm{cm}^{-1}$, $1232 \mathrm{~m} \mathrm{~cm}^{-1}, 1440 \mathrm{~m} \mathrm{~cm}^{-1}$, and $1463 \mathrm{~m} \mathrm{~cm}^{-1}$ and marked with an asterisk. c) Comparison of the SERS spectra obtained by UV-LA-SERS with that obtained by the standard colloid-based procedure. 
A sample taken from the red vest of the Virgin in the "Adoration of the Shepherds" by Giorgione (sample G) had already been analyzed in the past, both by applying a drop of silver colloid directly on the cross-section, and through VisLA-SERS. With the colloid technique we were able to identify the colorant in the red glaze, albeit with a weak signal. Vis-LA-SERS did not produce any peaks above the background, while significantly damaging the sample due to the relatively high penetration of the visible radiation in the sample.

As shown in Figure 4, UV-LA-SERS gave a good match with the kermesic acid reference. The superiority of UV-LASERS over the other techniques is then clearly shown by the better resolved spectrum, the low impact on the surface of the sample, and-in comparison to the use of colloids-the absence of any residues left over the surface.

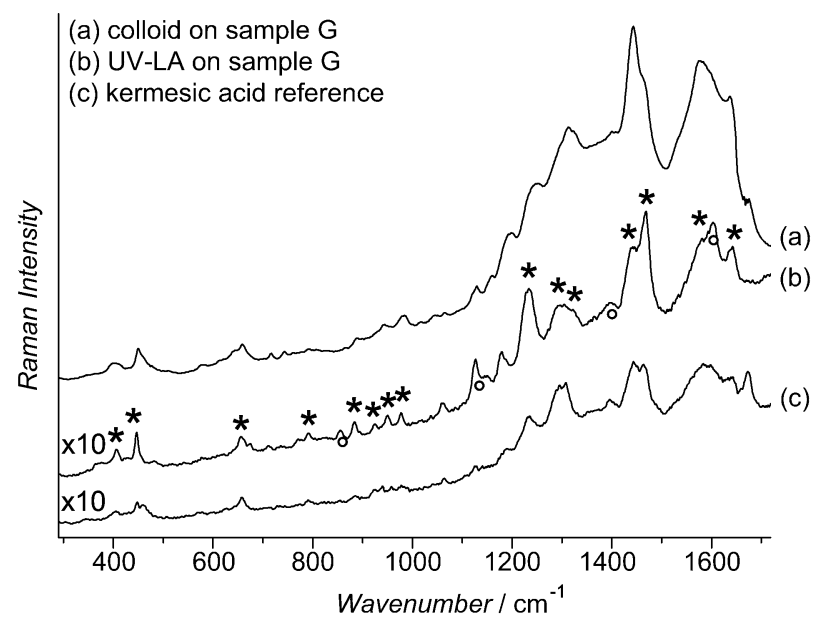

Figure 4. SERS spectra collected from the sample $G$ with the standard colloid technique and UV-LA-SERS. Signals of reference kermesic acid were detected at $405 \mathrm{w} \mathrm{cm}^{-1}, 447 \mathrm{~m} \mathrm{~cm}^{-1}, 459 \mathrm{w} \mathrm{cm}^{-1}, 790 \mathrm{vw} \mathrm{cm} \mathrm{cm}^{-1}$, $885 \mathrm{w} \mathrm{cm}^{-1}, 924 \mathrm{vw} \mathrm{cm} \mathrm{cm}^{-1}, 950 \mathrm{w} \mathrm{cm}^{-1}, 950 \mathrm{w} \mathrm{cm}^{-1}, 976 \mathrm{w} \mathrm{cm}^{-1}$,

$1232 \mathrm{~s} \mathrm{~cm}^{-1}, 1294 \mathrm{~m} \mathrm{~cm}^{-1}, 1308 \mathrm{~m} \mathrm{~cm}^{-1}, 1440 \mathrm{vs} \mathrm{cm}^{-1}, 1463 \mathrm{vs} \mathrm{cm}^{-1}$, $1579 \mathrm{~m} \mathrm{~cm}^{-1}, 1643 \mathrm{~m} \mathrm{~cm}^{-1}$ and marked with an asterisk.

In the case of the cross-sections from the Signorelli and the Giorgione paintings, UV-LA-SERS showed that the two Venetian masters used kermes, a dye that in a few decades would have been supplanted by cochineal coming from the New World. The use of the new technique allowed us to obtain this result without compromising the samples, which remain available for photography and further study by other complementary techniques.

It is worth noting that the achieved sensitivity and spatial resolution allow LA-SERS vibrational spectra to provide complimentary information to spot analysis mass spectrometry techniques of organic colorants, such as laser desorption ionization (LDI) and matrix-assisted laser desorption ionization (MALDI) mass spectrometry. We furthermore anticipate that the lack of a need for extraction solvents in our SERS approach will improve the ability to detect multiple species of colorants in a single measurement, though of course this would be limited by the complexity of the superimposed spectra and is unlikely to resolve mixtures as highly complex as what can be detected by separation techniques such as liquid chromatography and mass spectrometry. ${ }^{[12]}$

In conclusion, UV-LA-SERS provides a powerful new approach for organic pigment analysis, overcoming some of the drawbacks of traditional SERS techniques. The high spatial resolution and sensitivity of the method opens new frontiers in the study of cultural heritage material. In particular, the possibility to focus the analysis on single particles or on thin layer stratigraphy, with a minimum spot size in the range of $10 \mu \mathrm{m}$ extends the use of SERS to the analysis of historical painting layers, and in general, mixtures and complex, multilayered, and heterogeneous samples. The background signal can still be a problem, and future work will focus on creating a lower background SERS-active substrate and boosting the SERS signal. The approach has realistic potential for further increases in the spatial resolution up to $1 \mu \mathrm{m}$ with an optical setup improved for delivery of the UV laser pulse.

\section{Experimental Section}

The UV-LA-SERS instrument is based on a system described in detail elsewhere, ${ }^{[6,10]}$ and modified by adding a co-propagating $355 \mathrm{~nm}$ $\mathrm{Nd}$ :Yag laser beam ( $7 \mathrm{~ns}$ pulse, Opotek Opolette) and appropriate optics for the UV ablation of the sample. A $488 \mathrm{~nm}$ "read" laser (Evergreen Laser Corp.) can be aligned on the visible laser path by moving a flipping mirror. In this way, it is possible to ablate the sample and to record the SERS signal, without moving the vacuum chamber or breaking the vacuum. For the UV ablation a fluence of ca. $1 \mathrm{~J} \mathrm{~cm}^{-2}$ on the sample and three to four pulses were used as standard parameters for all the analysis here presented. The laser spot on the sample can be enlarged or made smaller by inserting or removing a quartz lens in the beam path, respectively, which translates the focal plane of the ablation laser.

The SERS-active substrate consists of a $10 \mathrm{~nm} \mathrm{Ag}$ nano-island film vapor deposited onto a quartz slide in a vacuum evaporator (Edwards E306A) at an evaporation rate of approximately $0.1 \AA^{-1}$. The window is placed upside down to seal the ablation chamber. The ablated plume solidifies directly on the SERS active substrate allowing the dye adsorption on it. The more detailed procedure and the properties of the film are described in previous works. ${ }^{\left[{ }^{6,11]}\right.}$

The SERS spectra reported here were obtained with a Bruker Senterra Raman instrument capable of a $3-5 \mathrm{~cm}^{-1}$ resolution. The sample was excited through a $488 \mathrm{~nm}$ solid state laser (Spectra Physics Cyan), with a power at the sample of about $0.4 \mathrm{~mW}$. The windows were removed from the chamber and all the spectra reported were acquired with a single integration of $30 \mathrm{~s}$, focusing the laser beam through a 20X long working distance microscope objective (Olympus LMPlanFL) on the silver substrate. For each sample, the SERS analysis was performed in the proximity of the area where the sample was adsorbed on the substrate and at progressively increasing distances.

The pretreatment step was obtained in a custom-made teflon chamber. A drop of HF is deposited in a polyethylene cap and left to vaporize. The cross-section is loaded into the chamber from a lateral aperture, sealed with a cap. The stopper of the chamber was drilled to allow the stream of HF vapor to flow over the sample. Before ablation the sample was degassed in a vacuum chamber.

Received: August 6, 2014

Published online: October 29, 2014 
[1] a) P. L. Stiles, J. Dieringer, N. C. Shah, R. P. Van Duyne, Annu. Rev. Anal. Chem. 2008, 1, 601-626; b) J. R. Lombardi, R. L. Birke, Acc. Chem. Res. 2009, 42, 734-742.

[2] a) M. V. Cañamares, J. V. Garcia-Ramos, C. Domingo, S. Sanchez-Cortes, J. Raman Spectrosc. 2004, 35, 921-927; b) M. V. Cañamares, M. Leona, M. Bouchard, C. M. Grzywacz, J. Wouters, K. Trentelman, J. Raman Spectrosc. 2010, 41, 391 397; c) A. V. Whitney, R. P. Van Duyne, F. Casadio, J. Raman Spectrosc. 2006, 37, 993-1002; d) K. Chen, M. Leona, K.-C. VoDinh, F. Yan, M. B. Wabuyele, T. Vo-Dinh, J. Raman Spectrosc. 2006, 37, 520-527; e) F. Casadio, M. Leona, J. R. Lombardi, R. Van Duyne, Acc. Chem. Res. 2010, 43, $782-791$.

[3] M. V. Cañamares, J. V. Garcia-Ramos, S. Sanchez-Cortes, M. Castillejo, M. Oujja, J. Colloid Interface Sci. 2008, 326, 103-109.

[4] B. Doherty, B. G. Brunetti, a. Sgamellotti, C. Miliani, J. Raman Spectrosc. 2011, 42, 1932-1938.
[5] K. Chen, K.-C. Vo-Dinh, F. Yan, M. B. Wabuyele, T. Vo-Dinh, Anal. Chim. Acta 2006, 569, 234-237.

[6] P. S. Londero, J. R. Lombardi, M. Leona, Anal. Chem. 2013, 85, $5463-5467$.

[7] D. Günther, B. Hattendorf, TrAC Trends Anal. Chem. 2005, 24, $255-265$.

[8] F. Pozzi, J. R. Lombardi, S. Bruni, M. Leona, Anal. Chem. 2012 , $84,3751-3757$

[9] see Ref. [2e].

[10] P. Londero, J. R. Lombardi, M. Leona, J. Raman Spectrosc. 2013, $44,131-135$

[11] M. Leona, J. Stenger, E. Ferloni, J. Raman Spectrosc. 2006, 37, $981-992$.

[12] I. Degano, E. Ribechini, F. Modugno, M. P. Colombini, Appl. Spectrosc. Rev. 2009, 44, 363-410. 